\title{
COVID-19: How to Disprove the Medical "Null Hypothesis" by the System of Systems Approach to Operant Conditioning of Social Behavioral Response
}

\author{
U Tuzun* \\ Alumnus Professor, University of Cambridge, United Kingdom
}

Submission: July 21, 2020; Published: August 06, 2020

*Corresponding author: U Tuzun, Alumnus Professor, University of Cambridge \& Professional Consultant, Weybridge, Surrey, United Kingdom

\begin{abstract}
Various task algorithm methodologies used to construct AI-Assisted Decision-Making Frameworks rely on the well-established rules of cognitive psychology development within a behavioral pattern recognition context. Analogies between the main drivers for the metacognitive learning processes in social behavioral environments and those that are implemented in mathematical codes for neural network construction and training are highlighted. The author has recently published works in these two seemingly disparate domains of intellectual endeavor only to recognize the formalism of the multitude of similarity rules that exist to facilitate a cognitive continuum from personal awareness-recognition to analysis-assessment and to synthesis-modification mirrored in both behavioral psychology and artificial intelligence domains. The importance of advancing null hypotheses and then construction of tasks to prove/disprove the "null state" by establishing statistically significant crosscorrelations require very large amounts of data gathering under different environmental conditions so as to boost the confidence intervals of the general validity of the conclusions. In neural network development, large data set convolutions are performed using unsupervised and unsupervised deep learning. In both cases, the causality patterns are established by reference to established "connectivity rules" which are predefined and rehearsed by controlled data modulations. The recent track and trace procedures used to gather statistically significant causality patterns of the spread of COVID-19 in various social environments by the asymptomatic carrier populations would do well to adhere to similar rules of data analysis and causality synthesis as advocated by the theoretical frameworks of neural network constructions and behavioral psychology patterns. A tentative framework will be presented as to how to implement such a framework for data gathering and analysis that can lead to scientifically robust findings.
\end{abstract}

Keywords: COVID-19; System of systems; Null hypothesis; Operant conditioning

Covid-19 Transmission Prevention: Social Behavior Modification and Physical Distancing

State-of-the-art scientific expertise is only able to recommend social behavior modification and physical isolation as the most effective preventative measures against the spread of Covid-19 by human-to-human transmission. Human populations, irrespective of their geographic locality and urban or rural habitation, are prescribed only one set of universal hygiene rules: wash your hands frequently at least for 20 seconds each time, avoid contact with face and eyes, cover your mouth and nose in public. This universal prescription of preventative rules of human social conduct does not recognize any differentiation by environmental factors such as size of social dwelling, number of co-habitants, local community density; or any individual physical attributes such as age, gender, ethnicity, weight, height, form of physical disability. This list is by no means complete and could be extended to cover more detailed specific aspects of social and physical differentiation depending upon the specific geographies and localities that can be evaluated. On top of the social behavioral restraints that are prescribed regarding the social habitation and hygiene, socio-economic activities are also curtailed by prescribing minimal use of public transport and working in isolation (e.g. use of home office) with minimal physical contact. Physical distancing in public and limitations on size of public gatherings are also prescribed with a view to minimize human-to-human transmission of the virus.

\section{Covid-19 Human Immunity Reinforcement: Universal}

\section{Vaccine Applications in Large Populations}

No effective medication could currently be prescribed which directly targets the virus in the human body. Malaria medication [1] and steroids [2] are found to have effect on reducing the 
mortality rate of those who are intensive care hospitalized. Against this background, all the intensive scientific effort and capital investment are focused on the development of different types of vaccines with the expectation that these would help to i) boost the human immune system and ii) allow the human body to develop antibodies to fight the virus. In vaccine research, the expectation is that vaccines developed will produce desired protective results universally with all human populations living different parts of the world. No tailor-made further modifications will be required to cater for any special environmental requirements or for different human physical attributes. The expectation projected by medical science, therefore, is that "one universal protection method" will work for all. This hypothesis is the famous "null hypothesis" which nullifies/minimizes the effects of differentiating factors on the causality of the virus and its transmission mechanisms. In the theory of probability statistics, the "null hypothesis" is also described as " the hypothesis that there is no significant difference between specified populations, any observed difference being made being exclusively due to sampling and experimental error"; see for example Kluger \& Tikochinsky [3] who demonstrate the limitations of the theoretical "null hypothesis" approach in reallife situations and Pampallona \& Tsiatis [4] who describe the one-sided and two-sided hypothesis testing of the theoretical null hypothesis.

\section{Use of Research Methods of Human Behavior and its applications to Covid-19}

At the time of this article, over 16 million have tested positive with Covid-19 with over 0.6 million deaths from the virus globally. The simple statistical calculation at the global scale yields a mortality rate of $4 \%$. National statistics produced for the World Health Organization, WHO in different countries shows very significant fluctuations of the mortality rate in different countries ranging from $2 \%-14 \%$. This very significant level of variation of the mortality rate in individual countries alone is sufficient to disprove the "null hypothesis" as described statistically above. Furthermore, these levels of statistical variance of the mortality rate can be normalized to show that from as little as $50 \%$ to over $300 \%$ variation of the global mean mortality rate as revealed by the individual national statistics declared so far. This simplest statistical normalization therefore indicates that as much as 6-fold or more variation is caused in virus-related deaths in different countries as recorded currently in the WHO databases. Clearly, against this background, science needs to question the root causes for these large variations. Furthermore, given that there is no universally accepted medication and cure for Covid-19 as yet and all the social-behavior modification prescriptions issued for the prevention of virus transmission are applied universally, what could be the systemic causes responsible for such large differentials in virus transmission and mortality rates?

To provide meaningful explanations to the above conundrum requires the use of robust scientific research methods. These can range from a) survey and test results to b) control group observations and to c) controlled scientific experiments. However, the application of any one of these methods in isolation is not likely to reveal answers of universal validity. The major weaknesses of isolated and singular research method applications are likely to include i) distorted results due to sampling errors and poorly phrased questions and response biases in a) above with ii) loss of experimental control and lack of random assignment associated with b) and the lack of robust mathematical frameworks to generalize to real world behavior associated with c) respectively.

Given the above weaknesses associated with the application of singular research methods in isolation, a more refined "system of systems" framework of increasing complexity is required where the neural connectivity between the cause and effect is diagnosed at different sub-levels between various components of the surrounding environment at different system boundaries and interaction interfaces and governing functions; see Figure 1 below.

Figure 1 features a) a specific investigated system with defined system boundaries and b) surrounding environmental systems and c) sub-system elements, components, relations, and interfaces defining the specific functional architecture of each individual surrounding system. The neural connectivity of multilevel causality in such a framework is defined by specific pattern recognition tests and preventive and corrective response task algorithms; see for further details, the recent publications by the author in student-centered active learning using socially-induced situate metacognition [5] and novel design of an AI-assisted multi-layer environmental perceptron for use in hybrid energy plants [6]. In the system of systems application depicted in the development of COVID-19 Immunity, 4 surrounding systems are identified all with mutual interactivity; refer to Figure 1. The two surrounding systems that contribute to the similarity in specific population groups' health condition and physical profile attributes are used to drive the approach to the so-called convergence to "herd immunity" whilst the social living and economic conditions are recognized as possible significantly divergent connections slowing down the attainment of the preferred state of wider population immunity. The details of the functional architectures of the individual surrounding systems are also to include capitallabor-service interfaces that contribute both to the functional quality and the efficiency of each individual surrounding system; see for example Timberlake [7].

Covid-19 Adverse Socio-Economic Intensity Suppression: Operant Psychological Conditioning of Social

\section{Behavior Patterns using Systems Methodology}

Medical science currently stipulates, not yet backed by hard evidence, that as much as $50 \%$ of an entire national population could be asymptomatic carriers and thus potential transmitters of the Covid-19 virus. It is further stipulated that a much higher percentage of the population in the 15-40 years age group 
could be asymptomatic carriers of the virus. This age group of course also makes up the majority of the active work force in a country whose activities directly support the national economy. Giving that a major exclusion of the young work force from the socio-economic activities to control the spread of the virus and minimize transmission would be disastrous to growth aspirations of national economies, an operant social-conditioning system based on the theory of behavioral psychology could be considered. Of course, any details of implementation by governance of such an approach will very much depend on the current political system attributes of a given country (i.e. surrounding system); refer to Figure 1 below.

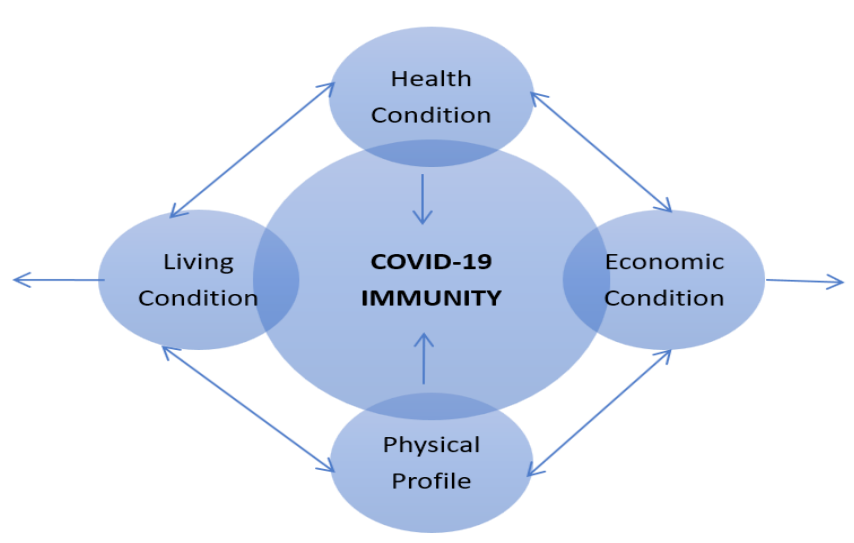

Figure 1: Human Behavioral System of Surrounding Systems Affecting COVID-19 Immunity i) Deterioration of living and economic conditions will impact adversely whilst improvements in health condition and physical profile will improve COVID-19 immunity, ii) All surrounding systems representing human condition are mutually interactive, iii) All surrounding systems have sub-systems and components that represent the functional architecture of each conditioning human response.

Active operant conditioning (see Timberlake [7] and Kirsch et al. [8]) recognizes a two-way system comprising of i) punishment to weaken/break the behavior pattern of undesirable consequences and ii) reward to reinforce behavior pattern yielding desirable consequences. A third way is also possible by passive operant conditioning, i.e. by consistently ignoring an undesirable behavior pattern with the expectation that no reinforcement may result in extinguishing it. The theory also recognizes positive and negative punisher as well as positive and negative reinforcer role models as possible alternatives to achieve desirable social conformity in human populations; refer to Figure 2 below.

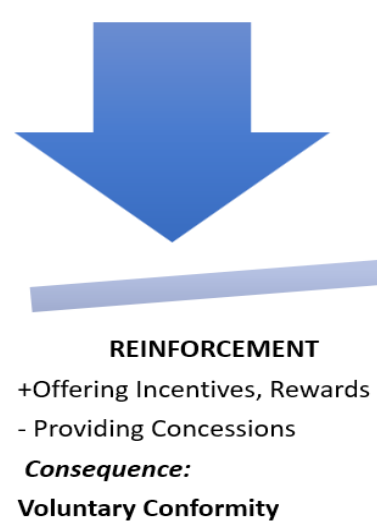

PUNISHMENT

+ Adding Tasks, Chores, Taxes

_ Restrictions, Withdrawing Priviliges

Consequence:

Compulsory Conformity

Figure 2: Operant Conditioning of Asymptomatic Covid-19 Carrier i) Surrounding system of national and local governance will affect the rate and intensity of impact on modifying human behavioral response, ii) cultural and religious factors will influence the type and extent of conformity, iii) asymptomatic human behavioral response will tilt to overwhelmingly voluntary mode with increasingly less punishment -reinforcement required when the surrounding systems affecting COVID-19 immunity are improved mutually.

For the operant conditioning system to work effectively, the subjects need to be well informed of a preferred behavior and the positive (rewards) or at least negative (concessions) reinforcement to be received when it is adopted [8]. Conversely, to stop the unwanted behavior, negative (withdrawing privileges) and positive (adding tasks) punishment modes could be introduced. 
Given the positive supportive environment needed to encourage conformity with preferred state of social behavior, it is highly unlikely that the governance will want to introduce measures that prioritize punishment over reinforcement. However, the lack of any clearly identified patterns of behavior and their consequential reinforcement (refer to Figure 2) by rewards and concessions is likely to yield to a confused state of conformity as opposed to the preferred state. It is believed that currently, a confused state of conformity with Covid-19 transmission prevention rules and regulations exists in most countries.

Social and economic priorities remain not defined clearly with little or no positive incentives apparent to encourage voluntary conformity of the populations with the new medically prudent and health conscious social living. On the other hand, the exercise of compulsory conformity by local/federal governance may increasingly result in cooperative social dissent if prolonged over lengthy periods and/or re-introduced with even tighter controls following periods of relaxation in response to future resurgence of virus transmissions and increases in mortality rates.

\section{Conclusion}

System of Systems approach described here is rooted in sound scientific principles with complementary mathematical frameworks that prove highly "transferable" in methodology to applications as diverse as engineering systems, metacognitive teaching and learning, and as demonstrated above, to modelling and conditioning socio-economic system behavioral responses to severe and recurrent infectious disease pandemics. Applications of the system of systems approach will require the continuing recognition of the significant variations of the individual country populations' disease transmission and mortality rates and the identification of patterns of causality between the specific environmental and human conditions together which constitute a cognitive platform for policy-driven and rule-based operant conditioning. This is also believed to disprove the "null hypothesis" favored by clinical science that barring sampling and experimental error, "one size fits all" approach is good enough for and in the development of therapeutic medicine to combat the virus. The application of the system methodology for reinforcementbased measures that can lead to voluntary conformity of the asymptomatic population groups will significantly ease the pressure on the identification and subsequent containment of the virus transmission.

\section{Acknowledgement}

The use of Oxford University, UK Centre for EvidenceBased Medicine (CEBM) digital communications is gratefully acknowledged.

\section{References}

1. Balfour H (2020) Accord to donate four million tablets to global COVID-19 Prophylaxis Trial. Multinational COPCOV Study News.

2. Armitage LC, Bretell R (2020) Inhaled Corticosteroids: A Rapid Review of the Evidence for Treatment or Prevention of COVID-19, June 22, Centre for Evidence-Based Medicine (CEBM), Nuffield Dept. of Primary Care Health Sciences, University of Oxford, United Kingdom.

3. Pampallona S, Tsiatis AA (1994) Group Sequential Designs for onesided and two-sided Hypothesis Testing with provision for early stopping in favour of the null hypothesis. Journal of Statistical Planning and Inference 42(1-2): 19-35.

4. Kluger AN, Tikochinsky J (2001) The error of accepting the "theoretical" null hypothesis: The rise, fall and resurrection of commonsense hypotheses in psychology. Psychological Bulletin American Psychological Association, (APA PsycNet), Washington DC, USA 127(3): 408-423.

5. Tuzun U (2020) Introduction to Systems Engineering and Sustainability Part I: Student-Centered Learning for Chemical and Biological Engineers. Education for Chemical Engineers 31: 85-93.

6. Tuzun U (2020) Artificial Intelligence Assisted Dynamic Control of Environmental Emissions from Hybrid Energy Process Plants (HEPP). Brief Research Report Frontiers in Energy Research 8: 179.

7. Timberlake W (1994) Behavior Systems, Associationism and Pavlovian Conditioning. Psychonomic Bulletin and Review 1(4): 405-420.

8. Kirsch I, Lynn SJ, Vigorito M (2004) Role of Cognition in Classical and Operant Conditioning, Journal of Clinical Psychology 60(4): 369-392.

\section{Your next submission with Juniper Publishers will reach you the below assets}

- Quality Editorial service

- Swift Peer Review

- Reprints availability

- E-prints Service

- Manuscript Podcast for convenient understanding

- Global attainment for your research

- Manuscript accessibility in different formats

( Pdf, E-pub, Full Text, Audio)

- Unceasing customer service

Track the below URL for one-step submission https://juniperpublishers.com/online-submission.php 